\title{
Digital LCR Instrument Oriented Wind Driven Power Control
}

\author{
Zhang weijia, Liu Wenxia, Sun shengli
}

Department of Application Arts and Science,Tianjin University of Science and Technology, City Tianjin, China

zhangweijia_tj@163.com

Keywords: distributed generation; grid-connected inverter; multi-mode switch; predictive power control

\begin{abstract}
With respect to the multi-state and multi-objective optimization problem of renewable energy grid-connection inverter system, this paper came up with a multi-mode predictive power control method to grid-connection inverter system, which established the discrete predictive model for island model and grid-connected model respectively and completed online self-matching of value function according to changes of system objective. The value function was established based on control objective that assure the quality of power supply voltage for local load under island power supply mode and indicated that the system switch would be optimal when minimum value of value function was taken. Besides, a rapid and accurate synchronous control method for power grid voltage was introduced to assure the smooth grid-connection and operation of inverter system. As for power-connected generation mode, control objective was converted to rapid and high accuracy tracking of system power. Finally, performance verification to control method proposed would be carried out based on 55kw grid-connected inverter prototype of renewable energy. The experiment results showed that the method could effectively improve the dynamic stability of grid-connected system and secure the electric energy quality at the access point of grid-connected system under island and power-connected mode.
\end{abstract}

\section{Introduction}

With the increasing seriousness of worldwide energy crisis, the development and utilization of renewable and clean energy are becoming a focus of concern gradually. Grid-connected inverter, as connecter to connect power grid and distributed generation, plays an important role in wind power generation, solar power generation and fuel cell power generation and even determines electric energy quality supplied by distributed power generation to some extent. Currently, the control strategy of mainstream grid-connected inverter comprises field oriented control (FOC) [6-7] and direct power control (DPC) [8-10]. FDC with linear PI regulator and PMI modulating cascade structure cannot secure the integrated optimization control of multi-mode grid-connected system. There are inherent disadvantages for DPC such as off-line complex planning and low-accuracy tracking and the like.

\section{System Modeling}

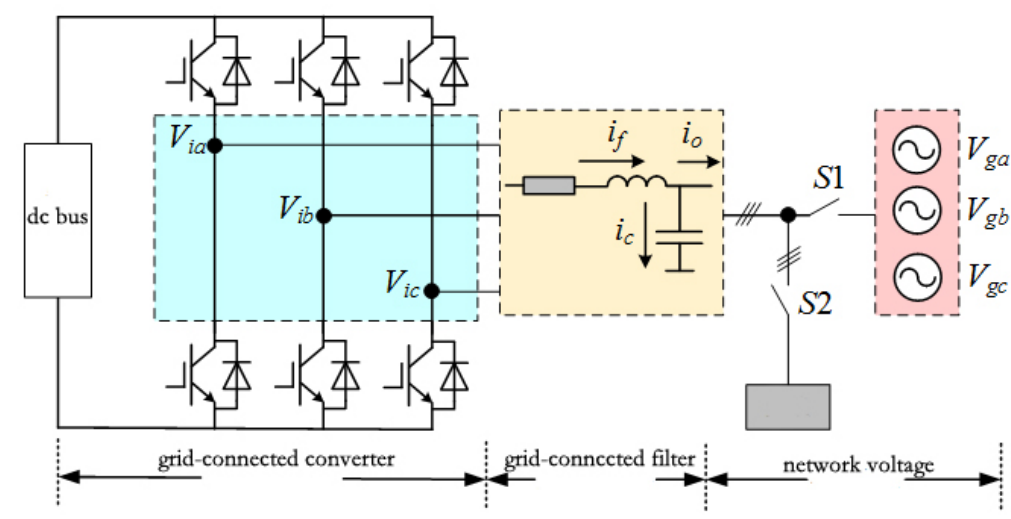

Fig.1 Diagram of renewable energy grid-connected inverter system 
As shown in figure 1, it is structure diagram of renewable energy three phase and two-level grid-connected inverter system. The system consists of grid-connected inverter, grid-connected filter and power grid voltage. Assume that three phase power grid voltage is symmetrical and sine and ignore switching loss and drop voltage of power switch device, 8 nos. voltage space vector outputted from two-level power-connected inverter is as:

$$
\boldsymbol{V}_{i}= \begin{cases}\frac{2}{3} V_{d c} e^{j(i-1) \frac{\pi}{3}} & (i=1 \cdots 6) \\ 0 & (i=0,7)\end{cases}
$$

\subsection{Island model}

When it is in island model, switch (S1) of main circuit is in on state and switch (S2) of by-pass circuit is in off state as shown in Figure 1. Renewable energy generation system keeps supplying power for adjacent circuit in power grid. At this moment, the dynamic model of LC filter energy-storage capacitor at inverter port is as:

$$
C \frac{d \boldsymbol{V}_{c}}{d t}=\boldsymbol{i}_{f}-\boldsymbol{i}_{L}
$$

In the formula, $C$ represents LC filter capacitor, $V c$ is vector to capacitor voltage, $i_{f}$ is vector of inductive current, $\boldsymbol{i}_{\mathrm{L}}$ is vector of load current. According to Kirchhoff's law, system space vector model is established as:

$$
\boldsymbol{V}_{i}=\boldsymbol{V}_{c}+\boldsymbol{i}_{f} R+L \frac{\boldsymbol{i}_{f}}{d t}
$$

In the formula, $\mathrm{L}$ is grid-side reactor and $\mathrm{R}$ is equivalent resistance.

\subsection{Grid-connected model}

When it is in power-connected running model, switch (S1) of main circuit is in off state and switch (S2) of by-pass circuit is in on state as shown in Figure 1. Renewable energy generation system carries out grid-connected generation through connection of input reactor and large power grid. At this moment, the inverter power-connected dynamic generation model is as:

$$
\begin{aligned}
& \boldsymbol{i}_{f}=\boldsymbol{i}_{o} \\
& \boldsymbol{i}_{o}=\boldsymbol{i}_{L}+\boldsymbol{i}_{g} \\
& \boldsymbol{V}_{i}=\boldsymbol{V}_{g}+\boldsymbol{i}_{o} R+L \frac{d \boldsymbol{i}_{o}}{d t}
\end{aligned}
$$

In the formula, $\boldsymbol{V}_{\mathrm{g}}$ represents vector to power grid voltage, $\boldsymbol{i}_{\mathrm{g}}$ is vector to power grid current, $\boldsymbol{i}_{\mathrm{o}}$ is vector to output current of inverter. According to instantaneous reactive power, instantaneous active power and reactive power of inverter is respectively as:

$$
\begin{aligned}
P & =\frac{3}{2} \operatorname{Re}\left\{\boldsymbol{V}_{g} \boldsymbol{i}_{o}^{*}\right\}=\frac{3}{2}\left(V_{g \alpha} i_{o \alpha}+V_{g \beta} i_{o \beta}\right) \\
Q & =\frac{3}{2} \operatorname{Im}\left\{\boldsymbol{V}_{g} \boldsymbol{i}_{o}^{*}\right\}=\frac{3}{2}\left(V_{g \beta} i_{o \alpha}-V_{g \alpha} i_{o \beta}\right)
\end{aligned}
$$

In the formula, * represents conjugate complex number, $V_{\mathrm{g} \alpha}$ and $V_{\mathrm{g} \beta}$ is real part and imaginary part of power grid voltage vector, $I_{\mathrm{g} \alpha}$ and $I_{\mathrm{g} \beta}$ is real part and imaginary part of output current vector.

\section{Voltage Control for Island Model}

When it is in island running model, grid-connected system needs to provide stable voltage for local load. At this moment, MPC controller shall predict the future trend of capacitor voltage vector and control it. According to formula (2) and formula (3), space system model is established as:

$$
\frac{d \boldsymbol{x}}{d t}=A \boldsymbol{x}=B \boldsymbol{V}_{i}+D \boldsymbol{i}_{L}
$$

In the formula: 


$$
\begin{aligned}
& x=\left[\begin{array}{l}
i_{f} \\
V_{c}
\end{array}\right], A=\left[\begin{array}{cc}
-R / L & -1 / L \\
1 / C & 0
\end{array}\right], B=\left[\begin{array}{c}
1 / L \\
0
\end{array}\right] \\
& D=\left[\begin{array}{c}
0 \\
-1 / C
\end{array}\right]
\end{aligned}
$$

Considering that control time of systematic sampling is small enough, the following assumption is made as:

$$
\begin{aligned}
e^{A T_{s}} & =1+A T_{s}+\frac{\left(A T_{s}\right)^{2}}{2 !}+\cdots+\frac{\left(A T_{s}\right)^{n}}{n !} \\
& \simeq 1+A T
\end{aligned}
$$

Now, discrete predictive model of LC filter capacitor voltage to grid-connected inverter system is established as:

$$
\boldsymbol{x}(k+1)=A_{\alpha} \boldsymbol{x}(k)+B_{b} V_{i}(k)+D_{d} i_{L}(k)
$$

In the formula:

$$
A_{\alpha}=e^{A T s}, B b=\int_{0}^{T s} e^{A t} d t, D_{d}=\int_{0}^{T s} e^{A t} D d t
$$

Therefore, the predictive value of $\mathrm{LC}$ filter capacitor voltage at $\mathrm{K}+1$ moment is as:

$$
\begin{aligned}
V_{c}(k+1)= & V_{c}(k)+e^{T_{S} / C} i_{f}(k)+\frac{C\left(e^{T_{s} / C}-1\right)}{L} V_{i}(k) \\
& -\frac{T_{s}}{C} i_{L}(k)
\end{aligned}
$$

To make sure that capacitor voltage tracks the change of expected value $\boldsymbol{V}_{\mathrm{c}}{ }^{*}$, value function $J_{\mathrm{V}}$ is established as:

$$
J_{V}=\left(V_{c \alpha}^{r e f}-V_{c \alpha}^{k+1}\right)^{2}+\left(V_{c \beta}^{r e f}-V_{c \beta}^{k+1}\right)^{2}
$$

In the formula, $V_{\mathrm{c} \alpha}{ }^{k+1}$ and $V_{\mathrm{c} \beta}{ }^{k+1}$ is real part and imaginary part of predictive value to capacitor voltage at $\mathrm{K}+1$ moment, as $V_{\mathrm{c}}(k+1)=V_{\mathrm{c} \alpha}{ }^{k+1}+j V_{\mathrm{c} \beta}{ }^{\mathrm{k}+1}$. According to formula 13, the voltage vector that value function $J_{\mathrm{V}}$ takes minimum value shall be used for the next system sampling period. The block diagram of voltage control for the island model is given in Figure 2.

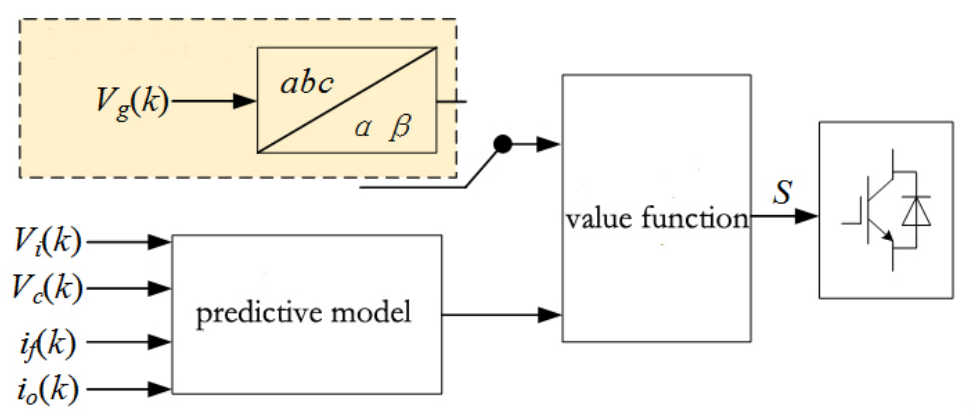

Fig.2 Block diagram of voltage control for the island model

\section{Experiment Verification}

The experiment results were shown in Figure 3 when it was synchronous switched to grid-connected model from the island model. These were from up to down as follows: capacitor voltage $V_{\text {ca }}$ to phase A and power grid voltage $V_{\text {ga }}$ to phase A and output current $i_{0}$ of inverter. As shown in Figure 3, starting from 0.1 second, it only took less than $1 \mathrm{~ms}$ for inverter output voltage to complete the synchronous match with power grid voltage. The amplitude, frequency and phase angle for both of them kept accurate consistency after 1.5 seconds upon completion of match. Furthermore, the capacitor voltage drop of LC filter could be ignored after 1.5 seconds. The whole process of voltage switch was smooth and there was no over current.

The experiment results on power adjustment under grid-connected inverter mode were shown in 
Figure 4. As seen in Figure, active power (P) carried out 20kw up and down adjustment at $0.22 \mathrm{~s}$ and $0.24 \mathrm{~s}$ respectively in which it responded rapidly and there was non-overshot. At the same time, reactive power always fluctuated around $0 \mathrm{kw}$ and it maintained good decoupling performance between $\mathrm{P}$ and $\mathrm{Q}$ in the whole process. Then, 20kVAar up and down experiments for reactive power (Q) were conducted at $0.26 \mathrm{~s}$ and 0.28 s respectively. It had the same effect with active power (P) experiment. It could be observed that reactive power (Q) responded rapidly and had a better decoupling effect than active power (P). By analyzing the inverter output current $i_{0}$, its amplitude and phase position conformed to the change law of $\mathrm{P}$ and $\mathrm{Q}$. And it kept better sine and there was no over current.
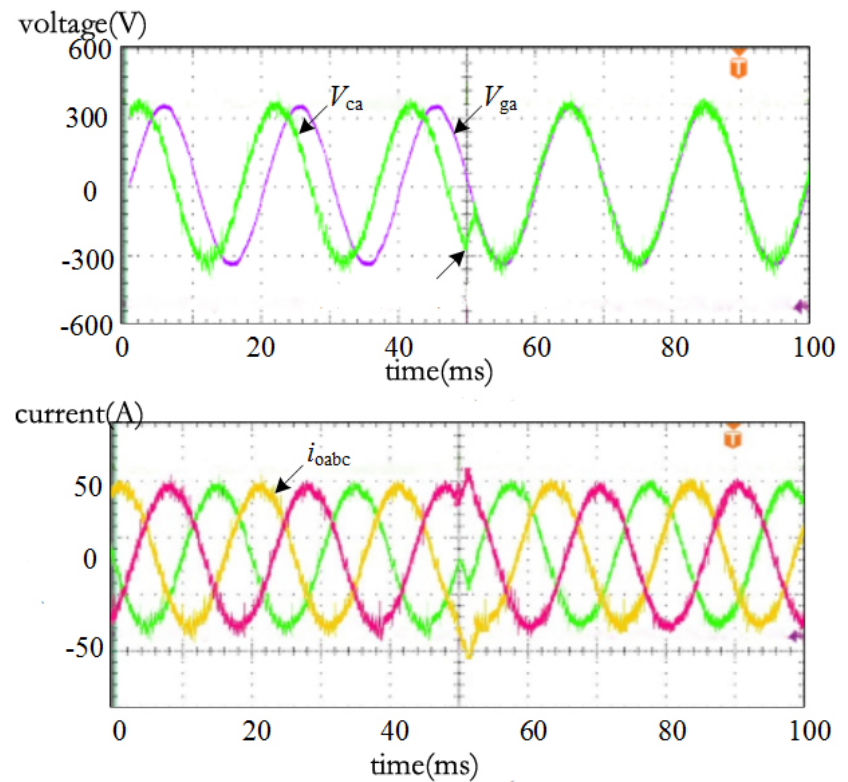

Fig.3 Dynamic experiment results
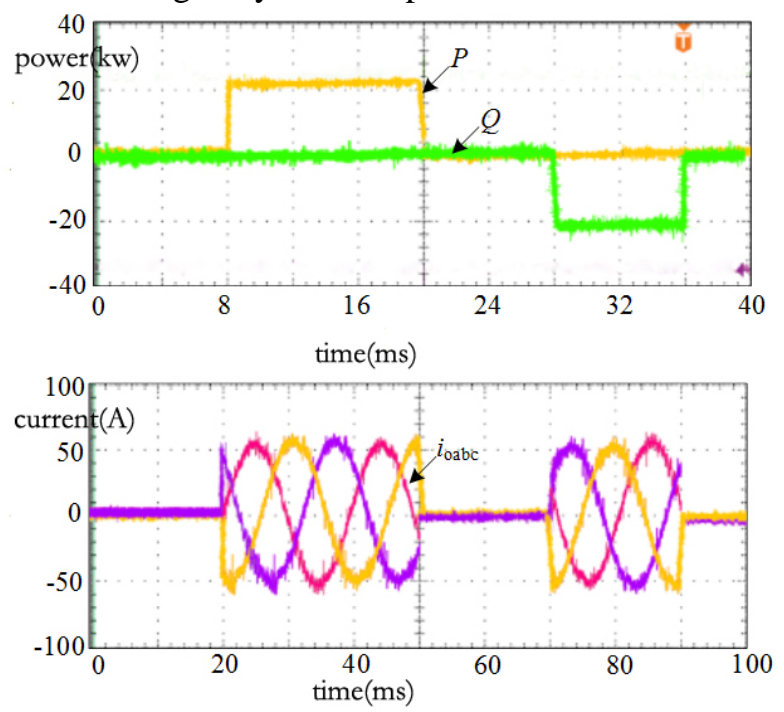

Fig4. Dynamic experiment results

\section{Conclusion}

In order to overcome the multi-state and multi-objective problems of renewable energy grid-connected inverter system, this paper came up with a multi-mode predictive power control method to grid-connection inverter system. The experiment results of $55 \mathrm{kv}$ prototype verified its feasibility and effectiveness. The following conclusion could be made: the method applying multi-mode predictive power control skill to renewable energy grid-connected inverter systematical control could effectively improve the dynamic stability of grid-connected system. The online switch mechanism to working state could achieve the free switch between the island generation mode and 
grid-connected inverter mode, which satisfied the requirement of grid-connected inverter system, solved the problem that the slow response arouse by oversize power in dynamic process and effectively extended grid-connected system to distributed generation system with complicated environment and changeable load.

\section{Acknowledge}

The Tianjin University of Science and Technology Foundation for scientific and research of China ,under Grant No.20130125

\section{References}

[1]Y. Geng, J. Chen, K. Pahlavan, Motion detection using RF signals for the first responder in emergency operations: A PHASER project, 2013 IEEE 24nd International Symposium on Personal Indoor and Mobile Radio Communications (PIMRC), London,Britain Sep. 2013

[2]Jie He, Yishuang Geng and Kaveh Pahlavan, Toward Accurate Human Tracking: Modelling Time-of-Arrival for Wireless Wearable Sensors in Multipath Environment, IEEE Sensor Journal, 14(11), 3996-4006, Nov. 2014

[3]Lv, Zhihan, Liangbing Feng, Haibo Li, and Shengzhong Feng. "Hand-free motion interaction on Google Glass." In SIGGRAPH Asia 2014 Mobile Graphics and Interactive Applications, p. 21. ACM, 2014.

[4]Li, Wubin, Johan Tordsson, and Erik Elmroth. "An aspect-oriented approach to consistency-preserving caching and compression of web service response messages." In Web Services (ICWS), 2010 IEEE International Conference on, pp. 526-533. IEEE, 2010.

[5]Y. Geng, J. He, K. Pahlavan, Modeling the Effect of Human Body on TOA Based Indoor Human Tracking[J], International Journal of Wireless Information Networks 20(4), 306-317 